\title{
Synthesis and transformations of some 1,2,4-trisubstituted pyrroles
}

\author{
Jarmila Štetinováa ${ }^{\mathrm{*}}$, Viktor Milata ${ }^{\mathrm{a}}$, Nad’a Prónayováb ${ }^{\mathrm{b}}$ Ognyan Petrov ${ }^{\mathrm{c}}$, \\ and Alexander Bartovič ${ }^{\mathbf{a}^{* *}}$ \\ ${ }^{a}$ Department of Organic Chemistry, ${ }^{b}$ Central Laboratory of Chemical Technics, \\ Faculty of Chemical and Food Technology, Slovak University of Technology, Radlinského 9, SK- \\ 81237 Bratislava, Slovak Republic \\ ${ }^{c}$ Department of Organic Chemical Technology, Faculty of Chemistry, University of Sofia, \\ BG-1126 Sofia, Bulgaria \\ E-mail: jarmila.stetinova@stuba.sk
}

Dedicated to Professor Lubor Fišera on his $60^{\text {th }}$ birthday

(received 16 Feb 05; accepted 23 Apr 05; published on the web 25 Apr 05)

\begin{abstract}
Alkyl 1-(2-alkoxy-2-oxoethyl)-4-formyl-1H-pyrrole-2-carboxylates 3a and $\mathbf{3 b}$ (alkyl = Me, Et), prepared from the corresponding alkyl 4-formyl-1H-pyrrole-2-carboxylates $\mathbf{2 a}$ and $\mathbf{2 b}$, have been modified to other derivatives of the same structural pattern. A series of $\beta$-pyrrolylalkenes 4-8 was obtained from pyrrole $\mathbf{3 b}$ and various $\mathrm{C}$-acids. Derivatives $\mathbf{2}$ and $\mathbf{3}$ have been also used as synthons for polysubstituted 4-(1H-pyrrol-3-yl)-1,4-dihydropyridines 9-12 under the conditions of the standard and the modified Hantzsch's dihydropyridine synthesis or by regioselective alkylation of the 1,4-dihydropyridine skeleton.
\end{abstract}

Keywords: Alkyl 1-(2-alkoxy-2-oxoethyl)-4-formyl-1H-pyrrole-2-carboxylates, $\beta$ pyrrolylalkenes, 4-(1H-pyrrol-3-yl)-1,4-dihydropyridines, regioselective alkylation

\section{Introduction}

Just like polysubstituted pyrroles, pyrrolecarboxylic acids and their derivatives also display a broad spectrum of bioactivity. Many of the latter compounds show appreciable antibacterial, ${ }^{1}$ and antitumor ${ }^{2}$ activity. Some of them are antibiotics, ${ }^{3}$ their suitably modified derivatives, ${ }^{4}$ effective anticonvulsants, ${ }^{5}$ and anti-inflammatory drugs. ${ }^{6}$ They can be used in the treatment of osseous diseases. ${ }^{7}$ The aim of this work is the synthesis of selected 1,2,4-trisubstituted pyrroles -

${ }^{\mathrm{a}^{* *}}$ present address: Synkola, Mlynská dolina, areál PvF UK, SK-842 15 Bratislava, Slovak Republic 
alkyl 1-(2-alkoxy-2-oxoethyl)-4-formyl-1H-pyrrole-2-carboxylates 3 - and their transformation to a series of $\beta$-pyrrolylalkenes 4-8 and polysubstituted 4-(1H-pyrrol-3-yl)-1,4-dihydropyridines 9-12. The specific biological testing of novel derivatives is planned. The alkenes 4-8 are in fact the substituted C-vinylpyrroles - extensively studied building blocks, widely employed in the synthesis of diverse representatives the pyrrole family, especially condensed heterocycles. ${ }^{8}$

\section{Results and Discussion}

The synthesis of compounds of the general formula 3 (Scheme 1) starts from pyrrole and proceeds via 2,2,2-trichloro-1-(1H-pyrrol-2-yl)ethanone and its alcoholysis to the alkyl $1 H$ pyrrole-2-carboxylates $\mathbf{1},{ }^{9,10}$ followed by the selective Friedel-Crafts formylation to the position 4 of the pyrrole ring. ${ }^{11,12}$ The formyl esters $\mathbf{2 a}$ and $\mathbf{2 b}$ were finally regioselectively alkylated at nitrogen atom with alkyl bromoacetates in the presence of potassium tert-butoxide and tetrabutylammonium bromide in dimethylformamide furnishing the 1,2,4-trisubstituted pyrroles $\mathbf{3} \mathbf{a}^{13}$ and $\mathbf{3 b}$ (yields $86 \%$ and $67 \%$, respectively).

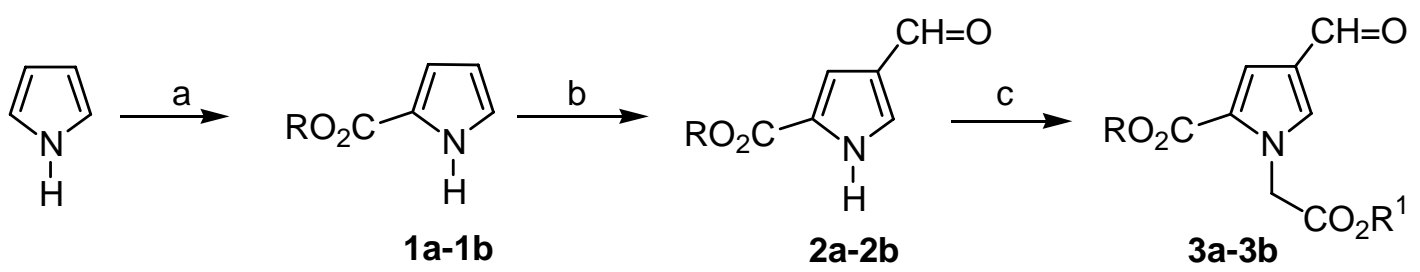

1a, 2a: $R=M e ; \quad$ 1b, 2b: $R=E t ; \quad 3 a: R=R^{1}=M e ; \quad 3 b: R=R^{1}=E t$

Scheme 1. Synthesis of alkyl 1-(2-alkoxy-2-oxoethyl)-4-formyl-1H-pyrrole-2-carboxylates 3 (a) 1. $\mathrm{CCl}_{3} \mathrm{COCl}, \mathrm{Et}_{2} \mathrm{O} ; 2$. RONa, ROH (R = Me, Et); (b) 1. $\mathrm{MeOCHCl}_{2}, \mathrm{AlCl}_{3}, \mathrm{MeNO}_{2}, \mathrm{CH}_{2} \mathrm{Cl}_{2}$, $1.5 \mathrm{~h} 0{ }^{\circ} \mathrm{C}, \mathrm{N}_{2} ; 2 . \mathrm{H}_{2} \mathrm{O}, \mathrm{CHCl}_{3}$; (c) $1 . \mathrm{BrCH}_{2} \mathrm{CO}_{2} \mathrm{R}^{1}\left(\mathrm{R}^{1}=\mathrm{Me}, \mathrm{Et}\right), \mathrm{Me}_{3} \mathrm{COK}, \mathrm{Bu}_{4} \mathrm{~N}^{+} \mathrm{Br}^{-}, \mathrm{DMF}$, $\mathrm{N}_{2}, 15-20(2 \mathrm{~h}) \rightarrow 40{ }^{\circ} \mathrm{C}(3 \mathrm{~h}) ; 2.2 \mathrm{H}_{2} \mathrm{O}, \mathrm{CHCl}_{3}$.

The structure of 1-alkylformylesters 3 as well as the following newly synthesized derivatives 4-12 was confirmed by elemental and spectral analysis data, mainly ${ }^{1} \mathrm{H}$ - and ${ }^{13} \mathrm{C}$ NMR data, in some cases supplemented by IR and MS spectra and x-ray analysis.

As in our own compounds $\mathbf{2 a}$ and $\mathbf{2 b}$, or those described in literature ${ }^{12}$ the ${ }^{1} \mathrm{H}$ NMR spectra of derivatives $\mathbf{3 a}$ and $\mathbf{3 b}$ showed higher chemical shift of 5-H protons of the pyrrole ring $(\delta 7.45-$ $7.92)$ than those of $3-\mathrm{H}$ protons $(\delta 7.26-7.39)$; the signals are doublets with the interaction constant $J=1.6-1.7 \mathrm{~Hz}$. Alkylation at nitrogen has been confirmed by the presence of the singlet of $\mathrm{N}-\mathrm{CH}_{2}$ at $\delta 5.09-5.20$, as well as by the presence of another alkyl group. The ${ }^{13} \mathrm{C}$ NMR spectral data of $\mathbf{3}$ correspond to the sugested structure. ${ }^{14}$ The x-ray analysis of the methyl ester 3a confirmed that both groups $\left(2-\mathrm{CO}_{2} \mathrm{CH}_{3}\right.$ and 4-CHO) interacted with the $\pi$-cloud of the 
pyrrole ring. The second methoxycarbonyl group at $N$ is twisted out of the pyrrole plane due to free rotation around the methylene group. ${ }^{13}$

In connection with the new data about the synthesis of C-vinylpyrroles, ${ }^{15}$ the series of substituted $\beta$-pyrolylalkenes 4-8 (Scheme 2) was obtained under the conditions of the Knoevenagel reaction of formyl ester $\mathbf{3 b}$ and various $\mathrm{C}$-acids, such as ethyl hydrogen malonate, ${ }^{16}$ diethyl malonate, (1,3-benzoxazol-2-yl)acetonitrile, ${ }^{17}$ (1,3-benzothiazol-2-yl)acetonitrile, ${ }^{18}(1 \mathrm{H}$ benzimidazol-2-yl)acetonitrile, ${ }^{19} \mathrm{~N}$-(1,3-benzothiazol-2-yl)cyanoacetamide, ${ }^{20 a}$ as well as its 6methoxyderivative, ${ }^{20 \mathrm{~b}}$ and malonodinitrile. Catalysed by piperidine, glycine, $10 \%$ ethanolic sodium ethoxide, and potassium acetate respectively, the reactions gave yields ranging from 50 $97 \%$.
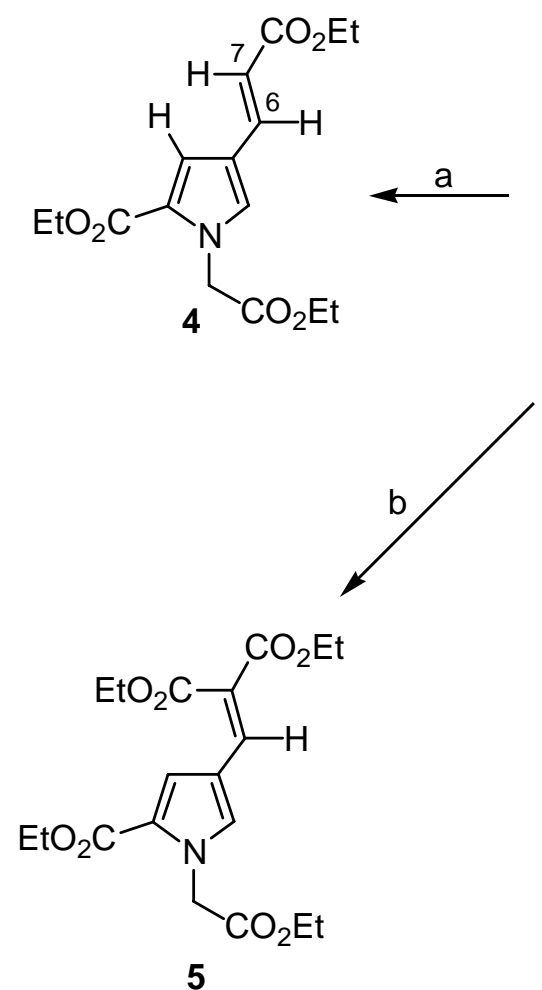
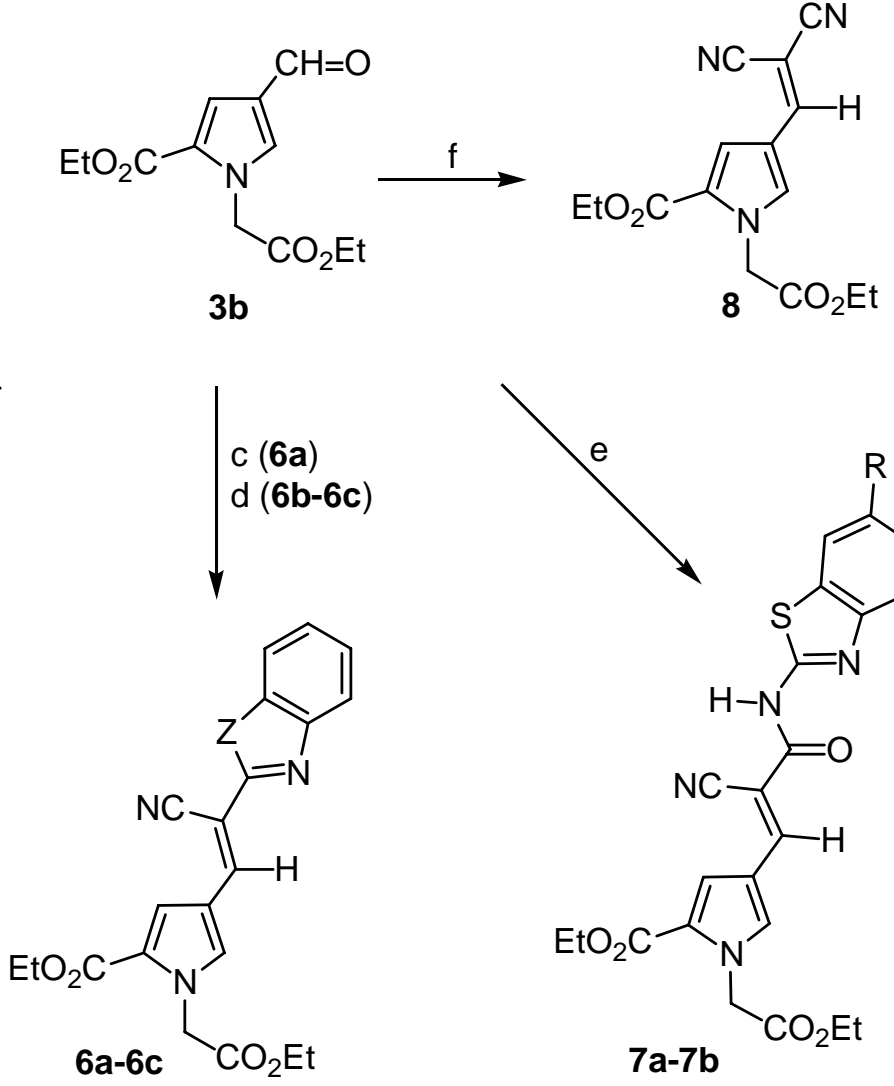

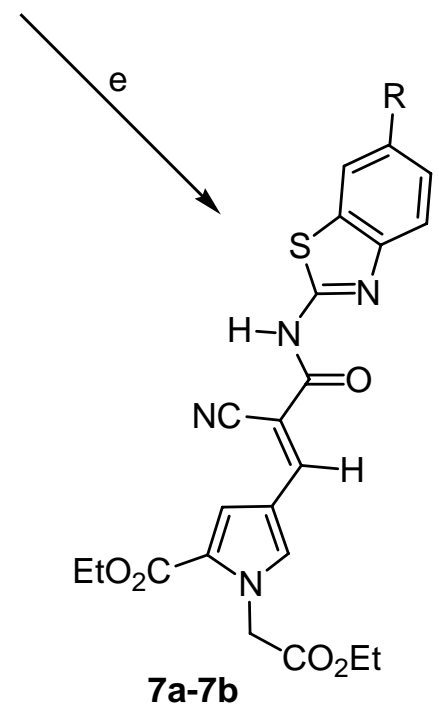

6a: $Z=O ; \quad 6 b: Z=S ; \quad 6 c: Z=N H ; 7 a: R=H ; 7 b: R=O M e$

Scheme 2. Synthesis of $\beta$-pyrrolylalkenes 4-8 (a) $\mathrm{CH}_{2}\left(\mathrm{CO}_{2} \mathrm{Et}\right) \mathrm{CO}_{2} \mathrm{H}$, pyridine, piperidine, $90{ }^{\circ} \mathrm{C}$, $24 \mathrm{~h}$; (b) $\mathrm{CH}_{2}\left(\mathrm{CO}_{2} \mathrm{Et}\right)_{2}$, piperidine, EtOH, reflux, $42 \mathrm{~h}$; (c) (1,3-benzoxazol-2-yl)acetonitrile, glycine, EtOH, reflux, $3 \mathrm{~h}$; (d) (1,3-benzothiazol-2-yl)- or (1H-benzimidazol-2-yl)acetonitrile, EtONa, EtOH, reflux, 30 min; (e) $N$-(1,3-benzothiazol-2-yl)- or $N$-(6-methoxy-1,3-benzothiazol2-yl)cyanoacetamide, AcOK, AcOH, reflux, 3h; (f) malonodinitrile, EtONa, EtOH, reflux, $1 \mathrm{~h}$. 
In compound 4 the interaction constant ${ }^{3} J(6-\mathrm{H}, 7-\mathrm{H})=15.6 \mathrm{~Hz}$ confirmed the $E$-arrangement of hydrogens at the newly formed multiple bond and the NOE DIF technique the mutual steric interaction of $3-\mathrm{H}$ and $7-\mathrm{H}$. Similarly, the coupling constant ${ }^{3} J(6-\mathrm{H}, \mathrm{CN})=13.1-14.1 \mathrm{~Hz}$ in compounds 6 and $7\left({ }^{13} \mathrm{C}\right.$ NOE technique) indicated the $E$-configuration of their substiutuents at the C-6-C-7 bond. The x-ray structural analysis of the derivative $6 \mathbf{b}(Z=S)$ manifested that both the benzothiazole and pyrrole ring were planar within the experimental error, and also approximately coplanar with plane of the ethylene bond (atoms $2^{\prime}$ - $\mathrm{C}, 7-\mathrm{C}, \mathrm{CN}, 6-\mathrm{C}, 6-\mathrm{H}, 4-\mathrm{C}$ ). ${ }^{21}$

Formyled derivatives 2 and $\mathbf{3}$ were further used for the preparation of polysubstituted 1,4dihydropyridines 9-12 (Scheme 3). In the area of dihydropyridine derivatives, which belong to notable antihypertensive drugs, ${ }^{22}$ only the synthesis of 4-(1H-pyrrol-2-yl)-1,4-dihydropyridines has been published, ${ }^{23}$ and their 3-pyrrolyl analogues remain unknown.

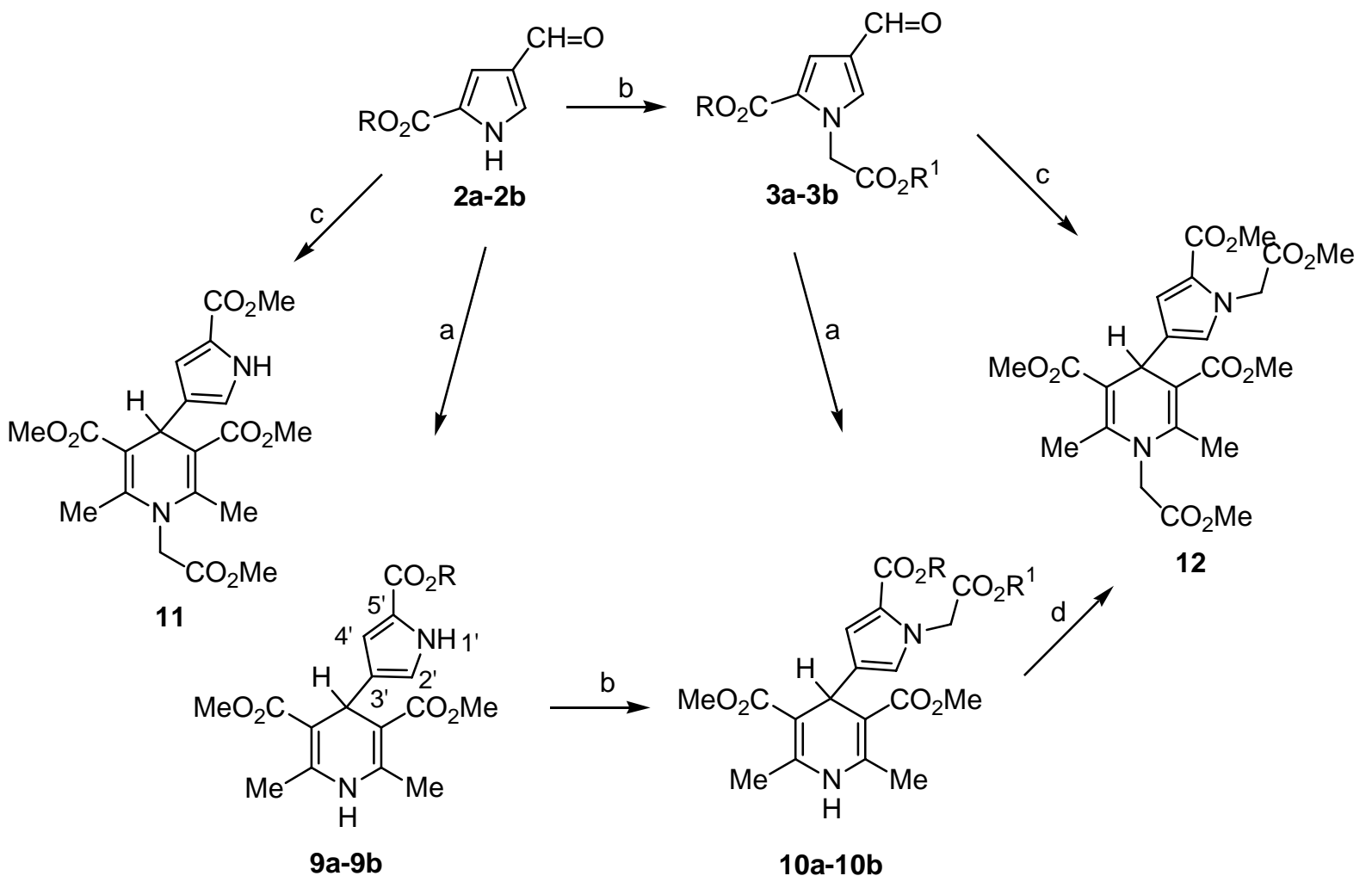

2a, 9a: $R=M e ; 2 b, 9 b: R=E t ; 3 a, 10 a: R=R^{1}=M e ; 3 b, 10 b: R=R^{1}=E t$

Scheme 3. Synthesis of 4-(1H-pyrrol-3-yl)-1,4-dihydropyridines 9-12 (a) $2 \mathrm{AcCH}_{2} \mathrm{CO}_{2} \mathrm{Me}$, $\mathrm{NH}_{4} \mathrm{OH}, \mathrm{MeOH}$; (b) $\mathrm{BrCH}_{2} \mathrm{CO}_{2} \mathrm{R}^{1}\left(\mathrm{R}^{1}=\mathrm{Me}\right.$, Et), $\mathrm{Me}_{3} \mathrm{COK}, \mathrm{Bu}_{4} \mathrm{~N}^{+} \mathrm{Br}^{-}, \mathrm{DMF}$; (c) 2 $\mathrm{AcCH}_{2} \mathrm{CO}_{2} \mathrm{Me}, \mathrm{H}_{2} \mathrm{NCH}_{2} \mathrm{CO}_{2} \mathrm{Me} . \mathrm{HCl}, \mathrm{MeONa}, \mathrm{MeOH}$, reflux, 10 h; (d) $\mathrm{BrCH}_{2} \mathrm{CO}_{2} \mathrm{Me}, \mathrm{K}_{2} \mathrm{CO}_{3}$, Aliquat 336 , closed tube, $200{ }^{\circ} \mathrm{C}, 4$ days. 
Dihydropyridines 9 and 10 were synthesized by the standard Hantzsch's method, ${ }^{24}$ involving refluxing of one equivalent of derivative 2 or 3 with two equivalents of methyl acetoacetate, and with an excess of ammonium hydroxide in methanol (Scheme 3, route a, yields 34-75\%).

1,4-Dihydropyridines 11 and 12, alkylated at nitrogen of the six-membered ring, were obtained by a modified Hantzsch's reaction, ${ }^{25}$ from formyl derivative $\mathbf{2 a}$ and $\mathbf{3 a}$ respectively, with methyl acetoacetate and glycin methyl ester, released in situ from its hydrochloride by the action of sodium methoxide in methanol (route c, yield 43-45\%).

A method of selective alkylation at both nitrogen atoms of the compound with general formula 9 was elaborated. Under the conditions leading to the alkylation of formyl esters $\mathbf{2}$, by increased concentration of alkylating agent (the ratio substrate : methyl bromoacetate was $1: 1.2$ and $1: 2.4$, respectively), derivatives $9 \mathbf{a}$ and $\mathbf{9 b}$ underwent alkylation only at pyrrole nitrogen, giving rise to the corresponding compounds $\mathbf{1 0 a}$ and $\mathbf{1 0 b}$ (route $b$, yield $43-45 \%$ ). Another alkylation of compound $\mathbf{1 0}$ at $N$ atom of 1,4-dihydropyridine could be achieved only under harsh conditions: solid-liquid phase-transfer catalysis without solvent ${ }^{26}$ in a large excess of methyl bromoacetate, $\mathrm{K}_{2} \mathrm{CO}_{3}$ and Aliquate 336 (Aldrich), heating at $200{ }^{\circ} \mathrm{C}$ in closed tube for 4 days (route $d$ ). Under such conditions compound 10a has been transformed to $\mathbf{1 2}$, identical with the compound obtained by route $c$ from $3 \mathbf{a}\left(\mathrm{R}=\mathrm{R}^{1}=\mathrm{Me}\right)$.

The ${ }^{1}$ H NMR spectra of compounds 9-12 showed the chemical shift of proton 4-H of the created 1,4-dihydropyridine ring in the range of $\delta$ 4.91-4.96. Both methyl and methoxycarbonyl groups were observed at $\delta 2.32-2.39(2,6-\mathrm{Me})$ and $3.69-3.72\left(3,5-\mathrm{CO}_{2} \mathrm{Me}\right)$, respectively. ${ }^{27}$ The signals of carbon atoms in the dihydropyridine nucleus of these derivatives were found in the range of $\delta 141.1-144.9$ (2,6-C), 102.9-106.6 (3,5-C), and 30.6-31.3 (4-C). ${ }^{27}$ The chemical shifts of the pyrrole ring carbons were designated with the help of calculations based on the published increments supposing that the effect of the dihydropyridine nucleus attached by its 3 position is similar to that of an isopropyl group. The derivatives 10a-10b showed the best agreement between the calculated and experimental data.

In conclusion, the preparation of $\beta$-pyrrolylalkenes 4-8 and 4-(1H-pyrrol-3-yl)-1,4dihydropyridines 9-12 utilizing alkyl 1-(2-alkoxy-2-oxoethyl)-4-formyl-1H-pyrrole-2carboxylates 3 (alkyl $=\mathrm{Me}, \mathrm{Et}$ ), converted from the corresponding alkyl $1 H$-pyrrole-2carboxylates 2 was demonstrated. Compounds 4-8 are the prospective synthons for new heterocycles, e. $g$. derivative 5 for distrontium salt of 3-(1-carboxymethyl-2-carboxypyrrol-4yl)pentanedioic acid (immobilization of osteoporosis). ${ }^{7}$

\section{Experimental Section}

General procedures. The temperature data are uncorrected. The melting points were determined on a Kofler hot plate apparatus. The IR spectra of $\mathrm{KBr}$ discs were recorded on a FTIR PU 9800 Philips spectrometer. The ${ }^{1} \mathrm{H}$ and ${ }^{13} \mathrm{C}$ NMR spectra were taken on a Bruker AC $250(250 \mathrm{MHz}$ for ${ }^{1} \mathrm{H}$ and $75 \mathrm{MHz}$ for ${ }^{13} \mathrm{C}$ ) in $\mathrm{CDCl}_{3}$ (TMS as internal reference) and on a Varian VXR-300 
spectrometer (299.945 MHz for ${ }^{1} \mathrm{H}$ and $75.429 \mathrm{MHz}$ for ${ }^{13} \mathrm{C}$ ) in DMSO- $d_{6}$ (chemical shift values were related to that of the solvent); APT, HETCOR, NOE and DIF NOE techniques were used for the assignments. The mass spectra (EI) were run with an MS 902 S (A. E. I. Manchester) spectrometer, equipped with direct inlet, electron energy of $70 \mathrm{eV}$, trap current $100 \mu \mathrm{A}$, ion source temperature $100-120{ }^{\circ} \mathrm{C}$ (for compounds $3 \mathbf{b}, \mathbf{4}, \mathbf{8}$ ) or $170-240{ }^{\circ} \mathrm{C}$ (for compounds 6 and 7).

Methyl $1 H$-pyrrole-2-carboxylate (1a), ${ }^{9}$ ethyl $\mathbf{1 H}$-pyrrole-2-carboxylate $(\mathbf{1 b}){ }^{10}$ methyl 4formyl-1H-pyrrole-2-carboxylate $(2 \mathrm{a}),{ }^{11}$ and ethyl 4-formyl-1H-pyrrole-2-carboxylate (2b), ${ }^{12}$ were prepared according to literature procedures.

Methyl 4-formyl-1-(2-methoxy-2-oxoethyl)-1H-pyrrole-2-carboxylate (3a). ${ }^{13}$ Yield $86 \%$. White needles, mp 70-3 ${ }^{\circ} \mathrm{C}$ (toluene / hexane 1:2). IR $v 1709,1676(\mathrm{C}=\mathrm{O}) \mathrm{cm}^{-1} ;{ }^{1} \mathrm{H} \mathrm{NMR}$ $\left(\mathrm{CDCl}_{3}\right) \delta 3.79\left(3 \mathrm{H}, \mathrm{s}, 1-\mathrm{CH}_{2} \mathrm{CO}_{2} \underline{\mathrm{Me}}\right), 3.83\left(3 \mathrm{H}, \mathrm{s}, 2-\mathrm{CO}_{2} \mathrm{Me}\right), 5.09\left(2 \mathrm{H}, \mathrm{s}, N-\mathrm{CH}_{2}\right), 7.39(1 \mathrm{H}, \mathrm{d}$, $J=1.7,3-\mathrm{H}), 7.45(\mathrm{~d}, 1 \mathrm{H}, J=1.7,5-\mathrm{H}), 9.78(1 \mathrm{H}, \mathrm{s}, \mathrm{CHO}) ;{ }^{13} \mathrm{C} \mathrm{NMR}\left(\mathrm{CDCl}_{3}\right) \delta 50.9(1-$ $\mathrm{CH}_{2} \mathrm{CO}_{2} \underline{\mathrm{Me}}$ ), $51.6\left(\mathrm{~N}-\mathrm{CH}_{2}\right), 52.5$ (2- $\mathrm{CO}_{2} \underline{\mathrm{Me}}$ ), 117.0 (3-C), 124.3 (4-C), 125.0 (2-C), 134.2 (5-C), 161.0 (2- $\left.\mathrm{CO}_{2} \mathrm{Me}\right), 168.1$ (1- $\left.\mathrm{CH}_{2} \underline{\mathrm{CO}}_{2} \mathrm{Me}\right), 185.3$ (CHO). Anal. Calcd. for $\mathrm{C}_{10} \mathrm{H}_{11} \mathrm{NO}_{5}$ (225.2): C, 53.33; H, 4.92; N, 6.22. Found: C, 53.23; H, 4.78; N, 6.08.

Ethyl 1-(2-ethoxy-2-oxoethyl)-4-formyl-1H-pyrrole-2-carboxylate (3b). Potassium tertbutoxide $(6.15 \mathrm{~g}, 55 \mathrm{mmol})$ and tetrabutylammonium bromide $(1.60 \mathrm{~g}, 5 \mathrm{mmol})$ in dimethylformamide $(20 \mathrm{~mL})$ were added to a stirred solution of ethyl 4-formyl-1H-pyrrole-2carboxylate $(\mathbf{2 b}, 8.36 \mathrm{~g}, 50 \mathrm{mmol})$ in the same solvent $(20 \mathrm{~mL})$ at $10-15{ }^{\circ} \mathrm{C}$, under nitrogen. A solution of ethyl bromoacetate $(9.2 \mathrm{~g}, 55 \mathrm{mmol})$ in dimethylformamide $(20 \mathrm{~mL})$ was added dropwise and the reraction mixture was stirred at $15-20{ }^{\circ} \mathrm{C}$ for $2 \mathrm{~h}$ and then at $40{ }^{\circ} \mathrm{C}$ for $3 \mathrm{~h}$. After evaporation in vacuo, a residue was treated with water $(80 \mathrm{~mL})$ and chloroform $(60 \mathrm{~mL})$. The combined organic layers were washed with water, $6 \mathrm{~N} \mathrm{HCl}(2 \times 30 \mathrm{~mL})$, again with water, dried (anh. $\mathrm{MgSO}_{4}$ ) and concentrated. After cooling, the oily product was treated with $n$-pentane (20-30 mL). The separated solid was recrystallized from $n$-hexane.Yield $8.4 \mathrm{~g}(67 \%)$, white crystals, mp 76-7 ${ }^{\circ} \mathrm{C}$. IR $v 1752,1686,1675(\mathrm{C}=\mathrm{O}) \mathrm{cm}^{-1} ;{ }^{1} \mathrm{H}$ NMR (DMSO- $\left.d_{6}\right) \delta 1.20,1.26(2 \mathrm{x}$ $\left.3 \mathrm{H}, \mathrm{t}, \mathrm{OCH}_{2} \underline{\mathrm{CH}}_{3}\right), 4.16,4.21\left(2 \times 2 \mathrm{H}, \mathrm{q}, \mathrm{OCH}_{2} \mathrm{CH}_{3}\right), 5.20\left(2 \mathrm{H}, \mathrm{s}, N-\mathrm{CH}_{2}\right), 7.26(1 \mathrm{H}, \mathrm{d}, J=1.6,3-$ $\mathrm{H}), 7.92(1 \mathrm{H}, \mathrm{d}, J=1.6,5-\mathrm{H}), 9.75(1 \mathrm{H}, \mathrm{s} \mathrm{CHO}) .{ }^{13} \mathrm{C} \mathrm{NMR}\left(\mathrm{DMSO}-d_{6}\right) \delta 14.0\left(\mathrm{CH}_{3}\right), 50.8(N-$ $\mathrm{CH}_{2}$ ), 60.3, 61.0 (2 x $\left.\mathrm{OCH}_{2}\right), 115.9$ (3-C), 124.2, 124.4 (2-C, 4-C), 135.9 (5-C), 160.0 (2$\left.\mathrm{CO}_{2} \mathrm{Et}\right), 168.1\left(1-\mathrm{CH}_{2} \mathrm{CO}_{2} \mathrm{Et}\right), 185.7$ (CHO); MS (EI) $\mathrm{M}^{+}, \mathrm{m} / z$ 253. Anal. Calcd. for $\mathrm{C}_{12} \mathrm{H}_{15} \mathrm{NO}_{5}$ (253.2): C, 56.91; H, 5.97; N, 5.53. Found: C, 56.49; H, 5.88; N, 5.56.

Ethyl-1-(2-ethoxy-2-oxoethyl)-4-[(1E)-3-ethoxy-3-oxoprop-1-enyl]-1H-pyrrole-2- carboxylate (4). A mixture of ethyl 1-(2-ethoxy-2-oxoethyl)-4-formyl-1H-pyrrole-2-carboxylate (3b, $2.53 \mathrm{~g}$, $10 \mathrm{mmol})$, ethyl hydrogen malonate $(1.32 \mathrm{~g}, 10 \mathrm{mmol}),{ }^{16}$ pyridine $(15 \mathrm{~mL})$ and piperidine $(0.5 \mathrm{~mL})$ was stirred at $90^{\circ} \mathrm{C}$ for $24 \mathrm{~h}$. After cooling and pouring on ice $(100 \mathrm{~g})$ and $\mathrm{HCl}(37 \%$, $22 \mathrm{~mL}$ ) mixture, the solid portion was filtered off and recrystallized from $n$-hexane. Yield $2.3 \mathrm{~g}$ (72\%), yellowish crystals, mp 73-4 ${ }^{\circ} \mathrm{C}$. IR $v 1760,1693(\mathrm{C}=\mathrm{O}), 1629(\mathrm{C}=\mathrm{C}) \mathrm{cm}^{-1}$; ${ }^{1} \mathrm{H}$ NMR ( DMSO-d $d_{6} \delta 1.19,1.23,1.25\left(3 \times 3 \mathrm{H}, \mathrm{t}, \mathrm{OCH}_{2} \underline{\mathrm{CH}}_{3}\right), 4.10-4.20\left(6 \mathrm{H}, \mathrm{m}, \mathrm{OCH}_{2} \mathrm{CH}_{3}\right), 5.08(2 \mathrm{H}, \mathrm{s}$, 
$\left.N-\mathrm{CH}_{2}\right), 6.28(1 \mathrm{H}, \mathrm{d}, J=15.6,7-\mathrm{H}), 7.29(1 \mathrm{H}, \mathrm{d}, J=1.6,3-\mathrm{H}), 7.50(1 \mathrm{H}, \mathrm{d}, J=15.6,6-\mathrm{H}), 7.54$ $(1 \mathrm{H}, \mathrm{d}, J=1.6,5-\mathrm{H}) ;{ }^{13} \mathrm{C} \mathrm{NMR}\left(\mathrm{DMSO}-d_{6}\right) \delta 14.0,14.1,14.2\left(3 \times \mathrm{CH}_{3}\right), 50.5\left(N-\mathrm{CH}_{2}\right), 59.6$, 60.0, $60.9\left(3 \times \mathrm{OCH}_{2}\right), 114.6$ (7-C), 115.7 (3-C), 118.9 (4-C), 123.6 (2-C), 132.5 (5-C), 137.5 (6C), 160.1 (2- $\left.\underline{\mathrm{CO}}_{2} \mathrm{Et}\right), 166.6$ (7- $\left.\underline{\mathrm{CO}}_{2} \mathrm{Et}\right), 168.4$ (1- $\left.\mathrm{CH}_{2} \mathrm{CO}_{2} \mathrm{Et}\right) ; \mathrm{MS}(\mathrm{EI}) \mathrm{M}^{+}, \mathrm{m} / z$ 323. Anal. Calcd. for $\mathrm{C}_{16} \mathrm{H}_{21} \mathrm{NO}_{6}(323.3)$ : C, 59.43; H, 6.55; N, 4.33. Found: C, 58.56; H, 6.41; N, 4.48. ${ }^{28}$

Diethyl $\{$ [5-(ethoxycarbonyl)-1-(2-ethoxy-2-oxoethyl)-1H-pyrrol-3yl]methylene\} malonate (5). Diethyl malonate $(3.20 \mathrm{~g}, 20 \mathrm{mmol})$ and piperidine $(1 \mathrm{~mL})$ were added to a solution of ethyl 1(2-ethoxy-2-oxoethyl)-4-formyl-1H-pyrrole-2- carboxylate (3b, $5.06 \mathrm{~g}, 20 \mathrm{mmol}$ ) in anhydrous ethanol $(100 \mathrm{~mL})$ and the mixture was stirred at reflux for $42 \mathrm{~h}$. After destillation off of solvent, the residue was treated with water $(80 \mathrm{~mL})$ and $\mathrm{CHCl}_{3}(50 \mathrm{~mL})$. The organic layer was separated and aqueous one was extracted with the same solvent $(3 \times 30 \mathrm{~mL})$. The combined chloroform solutions were washed with water, $6 \mathrm{~N} \mathrm{HCl}(2 \times 30 \mathrm{~mL})$, again with water, dried $\left(\mathrm{anh} . \mathrm{MgSO}_{4}\right)$ and evaporated to the dryness.Yield $7.55 \mathrm{~g}(95 \%)$, white crystals, mp $42-5{ }^{\circ} \mathrm{C}$ ( $n$-hexane). IR $v$ 1733, 1716, $1708(\mathrm{C}=\mathrm{O}), 1623(\mathrm{C}=\mathrm{C}) \mathrm{cm}^{-1}$; ${ }^{1} \mathrm{H}$ NMR (DMSO- $\left.d_{6}\right) \delta 1.17-1.30(12 \mathrm{H}, \mathrm{m}$, $\left.\mathrm{OCH}_{2} \underline{\mathrm{CH}}_{3}\right), 4.10-4.35\left(8 \mathrm{H}, \mathrm{m}, \mathrm{OCH}_{2} \mathrm{CH}_{3}\right), 5.14\left(2 \mathrm{H}, \mathrm{s}, N-\mathrm{CH}_{2}\right), 7.00(1 \mathrm{H}, \mathrm{d}, J=1.8,4-\mathrm{H}), 7.57$ $(1 \mathrm{H}, \mathrm{d}, J=1.8,2-\mathrm{H}), 7.57(1 \mathrm{H}, \mathrm{s}, 6-\mathrm{H}) ;{ }^{13} \mathrm{C} \mathrm{NMR}\left(\mathrm{DMSO}-d_{6}\right) \delta 13.7,14.0,14.1\left(4 \times \mathrm{OCH}_{2} \underline{\mathrm{CH}}_{3}\right)$, $50.6\left(\mathrm{~N}-\mathrm{CH}_{2}\right), 60.2,60.9,61.0,61.3\left(4 \times \mathrm{OCH}_{2} \mathrm{CH}_{3}\right), 116.0$ (3-C), 116.7 (4-C), 121.0 (7-C), 124.0 (5-C), 134.6 (2-C), 134.7 (6-C), 159.8 (5- $\left.\mathrm{CO}_{2} \mathrm{Et}\right), 163.9,166.5$ (2 x 7- $\left.\mathrm{CO}_{2} \mathrm{Et}\right), 168.3$ (1$\mathrm{CH}_{2} \mathrm{CO}_{2} \mathrm{Et}$ ); $\mathrm{MS}(\mathrm{EI}) \mathrm{M}^{+}, \mathrm{m} / z$ 395. Anal. Calcd. for $\mathrm{C}_{19} \mathrm{H}_{25} \mathrm{NO}_{8}$ (395.4): C, 57.71; $\mathrm{H}, 6.37$; , 3.54. Found: C, 57.55; H, 6.30; N, 3.66.

Ethyl 4-[(E)-2-(1,3-benzoxazol-2-yl)-2-cyanovinyl]-1-(2-ethoxy-2-oxoethyl)-1H-pyrrole-2carboxylate (6a). A mixture of (1,3-benzoxazol-2-yl)acetonitrile $(0.32 \mathrm{~g}, 2 \mathrm{mmol}),{ }^{17}$ ethyl 1-(2ethoxy-2-oxoethyl)-4-formyl-1H-pyrrole-2-carboxylate $(\mathbf{3 b}, 0.51 \mathrm{~g}, 2 \mathrm{mmol})$, and glycine $(0.1 \mathrm{~g}$, $1.35 \mathrm{mmol})$ was refluxed in ethanol $(30 \mathrm{~mL})$ for $3 \mathrm{~h}$. The solid, precipitating after cooling, was filtered off, washed thoroughly with water and dried. Yield $0.65 \mathrm{~g}(83 \%)$, yellow crystals, $\mathrm{mp}$ $168-9{ }^{\circ} \mathrm{C}$ (ethanol). ${ }^{1} \mathrm{H}$ NMR (DMSO- $\left.d_{6}\right) \delta 1.21,1.27\left(2 \times 3 \mathrm{H}, \mathrm{t}, \mathrm{OCH}_{2} \underline{\mathrm{CH}}_{3}\right), 4.17,4.24(2 \times 2 \mathrm{H}$, q, $\left.\mathrm{OCH}_{2} \mathrm{CH}_{3}\right), 5.24\left(2 \mathrm{H}, \mathrm{s}, N-\mathrm{CH}_{2}\right), 7.35-7.55\left(2 \mathrm{H}, \mathrm{m}, 5^{\prime}-\mathrm{H}, 6^{\prime}-\mathrm{H}\right), 7.69(1 \mathrm{H}, \mathrm{d}, J=1.8,3-\mathrm{H})$, 7.70-7.80 (2H, m, 4'-H, 7'-H), 8.03 (1H, d, $J=1.8,5-\mathrm{H}), 8.42$ (1H, s, 6-H); ${ }^{13} \mathrm{C}$ NMR (DMSO$\left.d_{6}\right) \delta 14.0,14.1\left(2 \mathrm{x} \mathrm{CH}_{3}\right), 50.9\left(\mathrm{~N}-\mathrm{CH}_{2}\right), 60.5,61.1\left(2 \times \mathrm{OCH}_{2}\right), 93.2(7-\mathrm{C}), 110.7$ (7'-C), 115.9 (CN), 116.4 (3-C), 117.2 (4-C), 119.7 (4'-C), 124.8 (2-C), 125.2 (6'-C), 125.8 (5'-C), 136.8 (5C), 141.2 (3'a-C), 143.3 (6-C), 150.0 (7'a-C), 159.2 (2'-C), 159.7 (2- CO $\left._{2} \mathrm{Et}\right), 168.1$ (1$\left.\mathrm{CH}_{2} \mathrm{CO}_{2} \mathrm{Et}\right) ;{ }^{3} J(6-\mathrm{H}, \mathrm{CN})=14.1$; MS (EI) M${ }^{+}, m / z$ 393. Anal. Calcd. for $\mathrm{C}_{21} \mathrm{H}_{19} \mathrm{~N}_{3} \mathrm{O}_{5}$ (393.4): C, 64.11; H, 4.87; N, 10.68. Found: C, 63.90; H, 4.83; N, 10.83 .

Ethyl 4-[(E)-2-(1,3-benzothiazol-2-yl)-2-cyanovinyl]-1-(2-ethoxy-2-oxoethyl)-1H-pyrrole-2carboxylate (6b). It was prepared from $\mathbf{3 b}$ and (1,3-benzothiazol-2-yl)acetonitrile ${ }^{18}$ according $^{-}$ the lit. ${ }^{21}$ Yield 51\%. Yellow crystals, mp $146-7{ }^{\circ} \mathrm{C}$ (ethanol). ${ }^{1} \mathrm{H}$ NMR (DMSO- $\left.d_{6}\right) \delta 1.21,1.27$ $\left(2 \times 3 \mathrm{H}, \mathrm{t}, \mathrm{OCH}_{2} \underline{\mathrm{CH}}_{3}\right), 4.17,4.24\left(2 \times 2 \mathrm{H}, \mathrm{q}, \mathrm{OCH}_{2} \mathrm{CH}_{3}\right), 5.24\left(2 \mathrm{H}, \mathrm{s}, N-\mathrm{CH}_{2}\right), 7.46(1 \mathrm{H}, \mathrm{t}, J=$ 7.6, 6'-H), $7.55\left(1 \mathrm{H}, \mathrm{t}, J=7.6,5^{\prime}-\mathrm{H}\right), 7.69(1 \mathrm{H}, \mathrm{s}, 3-\mathrm{H}), 7.97(1 \mathrm{H}, \mathrm{s}, 5-\mathrm{H}), 8.01(1 \mathrm{H}, \mathrm{d}, J=7.9$, $\left.4^{\prime}-\mathrm{H}\right), 8.12\left(1 \mathrm{H}, \mathrm{d}, J=7.9,7\right.$ '-H), $8.28(1 \mathrm{H}, \mathrm{s}, 6-\mathrm{H}) ;{ }^{13} \mathrm{C}$ NMR (DMSO- $\left.d_{6}\right) \delta 14.0,14.1 \quad(2 \mathrm{x}$ $\left.\mathrm{CH}_{3}\right), 50.9\left(\mathrm{~N}-\mathrm{CH}_{2}\right), 60.5,61.1\left(2 \times \mathrm{OCH}_{2}\right), 100.3$ (7-C), 116.4 (3-C), 116.8 (CN), 117.2 (4-C), 
122.3 (4'-C), 122.7 (7’-C), 124.6 (2-C), 125.8 (6'-C), 126.9 (5'-C), 133.9 (7'a-C), 136.4 (5-C), 141.8 (6-C), 152.9 (3'a-C), 159.7 (2- $\left.\mathrm{CO}_{2} \mathrm{Et}\right), 163.5$ (2'-C), $168.1\left(1-\mathrm{CH}_{2} \mathrm{CO}_{2} \mathrm{Et}\right) ;{ }^{3} J(6-\mathrm{H}, \mathrm{CN})=$ 14.1; MS (EI) $\mathrm{M}^{+}, m / z$ 409. Anal. Calcd. for $\mathrm{C}_{21} \mathrm{H}_{19} \mathrm{~N}_{3} \mathrm{O}_{4} \mathrm{~S}$ (409.4): C, 61.60; H, 4.68; N, 10.26; S, 7.83. Found: C, 61.63; H, 4.85; N, 10.52; S, 8.15.

Ethyl4-[(E)-2-(1H-benzimidazol-2-yl)-2-cyanovinyl]-1-(2-ethoxy-2-oxoethyl)-1H- pyrrole 2-carboxylate (6c). A solution of ethyl 1-(2-ethoxy-2-oxoethyl)-4-formyl-1H-pyrrole-2carboxylate $(\mathbf{3 b}, 0.51 \mathrm{~g}, 2 \mathrm{mmol}) \quad$ in ethanol $(10 \mathrm{~mL})$ and $10 \%$ ethanolic sodium ethoxide (3-4 drops) were added to a stirred solution of (1H-benzimidazol-2-yl)acetonitrile $(0.31 \mathrm{~g}, 2 \mathrm{mmol})^{19}$ in the same solvent $(10 \mathrm{~mL})$. After $30 \mathrm{~min}$ at reflux, the reaction mixture was gradually cooled to room $\mathrm{T}$ with stirring. The precipitate was filtered off and recrystallized from ethanol. Yield $0.4 \mathrm{~g}$ (51\%), yellow crystals, mp 241-3 ${ }^{\circ} \mathrm{C}$. IR $v 2224(\mathrm{CN}), 1754,1692(\mathrm{C}=\mathrm{O}), 1626(\mathrm{C}=\mathrm{C}) \mathrm{cm}^{-1} ;{ }^{1} \mathrm{H}$ NMR (DMSO-d $d_{6} \delta 1.20,1.27\left(2 \times 3 \mathrm{H}, \mathrm{t}, \mathrm{OCH}_{2} \underline{\mathrm{CH}}_{3}\right), 4.16,4.23\left(2 \times 2 \mathrm{H}, \mathrm{q}, \mathrm{OCH}_{2} \mathrm{CH}_{3}\right), 5.23$ $\left(2 \mathrm{H}, \mathrm{s}, N-\mathrm{CH}_{2}\right), 7.17-7.25\left(2 \mathrm{H}, \mathrm{m}, 5^{\prime}-\mathrm{H}, 6{ }^{\prime}-\mathrm{H}\right), 7.50-7.64\left(\mathrm{~m}, 4^{\prime}-\mathrm{H}, 7^{\prime}-\mathrm{H}\right), 7.66(1 \mathrm{H}, \mathrm{d}, J=1.8$, 3-H), $7.85(1 \mathrm{H}, \mathrm{d}, J=1.8,5-\mathrm{H}), 8.18(1 \mathrm{H}, \mathrm{s} 6-\mathrm{H}), 12.9(1 \mathrm{H}, \mathrm{bs}, \mathrm{NH}) ;{ }^{13} \mathrm{C}$ NMR (DMSO- $\left.d_{6}\right) \delta$ 14.0, $14.2\left(2 \times \mathrm{CH}_{3}\right), 50.7\left(\mathrm{~N}-\mathrm{CH}_{2}\right), 60.4,61.8\left(2 \times \mathrm{OCH}_{2}\right), 97.3$ (7-C), 115.7 (3-C, 4'-C, 7'-C), 117.1 (CN), 117.4 (4-C), 122.6 (5'-C, 6'-C), 124.5 (2-C), 132.5 (3'a-C, 7'a-C), 135.3 (5-C), 138.8 (6-C), 147.9 (2'-C), 159.8 (2- $\left.\mathrm{CO}_{2} \mathrm{Et}\right), 168.2\left(1-\mathrm{CH}_{2} \mathrm{CO}_{2} \mathrm{Et}\right) ;{ }^{3} J(6-\mathrm{H}, \mathrm{CN})=14.1$; MS (EI) $\mathrm{M}^{+}, m / z$ 392. Anal. Calcd. for $\mathrm{C}_{21} \mathrm{H}_{20} \mathrm{~N}_{4} \mathrm{O}_{4}$ (392.4): C, 64.27; H, 5.14; N, 14.28. Found: C, 63.65; $\mathrm{H}, 4.96 ; \mathrm{N}, 14.41$.

Ethyl 4-[(1E)-3-(1,3-benzothiazol-2-ylamino)-2-cyano-3-oxoprop-1-enyl]-1-(2-ethoxy2-oxoethyl)-1H-pyrrole-2-carboxylate (7a). A mixture of ethyl 1-(2-ethoxy-2-oxoethyl)-4formyl-1H-pyrrole-2-carboxylate $\quad(3 \mathbf{b}, \quad 0.51 \mathrm{~g}, \quad 2 \mathrm{mmol}), \quad N-\quad$ (1,3-benzothiazol-2yl)cyanoacetamide $(0.43 \mathrm{~g}, 2 \mathrm{mmol}){ }^{20 \mathrm{a}}$ anh. potassium acetate $(0.5 \mathrm{~g}, 5 \mathrm{mmol})$, and acetic acid $(15 \mathrm{~mL})$ was stirred while refluxing for $3 \mathrm{~h}$. After pouring on ice, the raw product was collected by filtration, washed with water, and dried. Yield $0.75 \mathrm{~g}(83 \%)$. Yellow crystals, $\mathrm{mp} 222-3{ }^{\circ} \mathrm{C}$ (acetic acid). ${ }^{1} \mathrm{H}$ NMR (DMSO-d $d_{6} \delta 1.22,1.28\left(2 \times 3 \mathrm{H}, \mathrm{t}, \mathrm{OCH}_{2} \underline{\mathrm{CH}}_{3}\right), 4.10-4.30(2 \times 2 \mathrm{H}, \mathrm{m}$, $\left.\mathrm{OCH}_{2} \mathrm{CH}_{3}\right), 5.22\left(1 \mathrm{H}, \mathrm{s}, N-\mathrm{CH}_{2}\right), 7.31\left(1 \mathrm{H}, \mathrm{t}, J=7.6,6^{\prime}-\mathrm{H}\right), 7.45\left(1 \mathrm{H}, \mathrm{t}, J=7.6,5^{\prime}-\mathrm{H}\right), 7.52(1 \mathrm{H}$, $\left.\mathrm{d}, J=8.2,4^{\prime}-\mathrm{H}\right), 7.66(1 \mathrm{H}, \mathrm{d}, J=1.8,3-\mathrm{H}), 7.90-8.00(2 \mathrm{H}, \mathrm{m}, 5-\mathrm{H}, 7$ '-H), 8.37 (1H, s, 6-H), $12.3(1 \mathrm{H}, \mathrm{bs}, \mathrm{NH}) ;{ }^{13} \mathrm{C}$ NMR $\left(\mathrm{DMSO}-d_{6}\right) \delta 14.2,14.3\left(2 \mathrm{x} \mathrm{CH}_{3}\right), 51.1\left(\mathrm{~N}-\mathrm{CH}_{2}\right), 60.7,61.3(2 \mathrm{x}$ $\mathrm{OCH}_{2}$ ), 102.6 (7-C), 116.7 (3-C), 117.0 (4-C), 117.5 (CN), 122.6 (4'-C), 124.0 (7'-C), 125.0 (2C), 127.0 (5'-C, 6'-C), 129.3 (7'a-C), 137.2 (5-C), 142.0 (3'a-C), 145.9 (6-C), 159.9 (2'-C, 2$\left.\mathrm{CO}_{2} \mathrm{Et}\right), 166.0(\mathrm{C}=\mathrm{O}) ;{ }^{3} J(6-\mathrm{H}, \mathrm{CN})=13.1$; MS (EI) $\mathrm{M}^{+}, m / z$ 452. Anal. Calcd. for $\mathrm{C}_{22} \mathrm{H}_{20} \mathrm{~N}_{4} \mathrm{O}_{5} \mathrm{~S}$ (452.5): C, 58.39; H, 4.46; N, 12.38. Found: C, 57.95; H, 4.75; N, 11.98.

Ethyl 4-[(1E)-2-cyano-3-(6-methoxy-1,3-benzothiazol-2-ylamino)-3-oxoprop-1-enyl]-1-(2ethoxy-2-oxoethyl)-1H-pyrrole-2-carboxylate (7b). It was prepared from $\mathbf{3 b}$ and $\mathrm{N}$-(6methoxy-1,3-benzothiazol-2-yl)cyanoacetamide ${ }^{20 \mathrm{~b}}$ according to procedure for $7 \mathbf{a}$. Yield 93\%, yellow crystals, mp $229-31{ }^{\circ} \mathrm{C}$ (ethanol). ${ }^{1} \mathrm{H}$ NMR (DMSO- $\left.d_{6}\right) \delta 1.21,1.27\left(2 \times 3 \mathrm{H}, \mathrm{t}, \mathrm{CH}_{2} \underline{\mathrm{CH}}_{3}\right)$, $3.81\left(3 \mathrm{H}, \mathrm{s} \mathrm{OCH}_{3}\right), 4.08-4.30\left(2 \times 2 \mathrm{H}, \mathrm{m}, \mathrm{OCH}_{2} \mathrm{CH}_{3}\right), 5.24\left(2 \mathrm{H}, \mathrm{s}, N-\mathrm{CH}_{2}\right), 7.06(1 \mathrm{H}, \mathrm{dd}, J=$ $\left.8.8, J=2.5,5^{\prime}-\mathrm{H}\right), 7.50-7.61\left(2 \mathrm{H}, \mathrm{m}, 4^{\prime}-\mathrm{H}, 7^{\prime}-\mathrm{H}\right), 7.67(1 \mathrm{H}, \mathrm{d}, J=1.6,3-\mathrm{H}), 7.93(1 \mathrm{H}, \mathrm{d}, J=$ 1.6, 5-H), $8.37(1 \mathrm{H}, \mathrm{s}, 6-\mathrm{H}), 13.00(1 \mathrm{H}, \mathrm{bs}, \mathrm{NH}),{ }^{13} \mathrm{C} \mathrm{NMR}\left(\mathrm{DMSO}-d_{6}\right) \delta 14.0,14.1\left(2 \times \mathrm{CH}_{3}\right)$, 
$50.9\left(\mathrm{~N}-\mathrm{CH}_{2}\right), 55.6\left(\mathrm{OCH}_{3}\right), 60.5,61.1\left(2 \times \mathrm{OCH}_{2}\right), 102.6(7-\mathrm{C}), 105.4$ (7'-C), 115.2 (5'-C), 116.4 (3-C, 4'-C), 116.8 (4-C), 117.2 (CN), 124.8 (2-C), 131.0 (7'a-C), 136.9 (5-C), 140.5 (3'aC), 145.5 (6-C), 156.3 (6'-C), 159.7 (2'-C, 2- $\left.\mathrm{CO}_{2} \mathrm{Et}\right), 165.5(\mathrm{C}=\mathrm{O}), 168.1\left(1-\mathrm{CH}_{2} \mathrm{CO}_{2} \mathrm{Et}\right){ }^{3} J(6-$ $\mathrm{H}, \mathrm{CN})=13.6$; MS (EI) $\mathrm{M}^{+}, m / z$ 482. Anal. Calcd. for $\mathrm{C}_{23} \mathrm{H}_{22} \mathrm{~N}_{4} \mathrm{O}_{6} \mathrm{~S}$ (482.5): C, 57.25; $\mathrm{H}, 4.60$; N, 11.61. Found: C, 57.12; H, 4.86; N, 11.84.

Ethyl 4-(2,2-dicyanovinyl)-1-(2-ethoxy-2-oxoethyl)-1H-pyrrole-2-carboxylate (8). A solution of ethyl 1-(2-ethoxy-2-oxoethyl)-4-formyl-1H-pyrrole-2-carboxylate (3b, $0.51 \mathrm{~g}, 2 \mathrm{mmol})$ in ethanol $(20 \mathrm{~mL})$ and $15 \%$ ethanolic natrium ethanolate (3-4 drops) were added to a hot solution of malonodinitrile $(0.154 \mathrm{~g}, 2.2 \mathrm{mmol})$ in the same solvent $(10 \mathrm{~mL})$. After stirring at reflux for 1 $\mathrm{h}$, the reaction mixture was treated with charcoal, filtered and cooled. The separated solid was filtered off. Yield $0.3 \mathrm{~g}$ (50\%), white needles, mp 124-5 ${ }^{\circ} \mathrm{C}$ (ethanol). IR $v 2227$ (CN), 1744, $1691(\mathrm{C}=\mathrm{O}), \mathrm{cm}^{-1} ;{ }^{1} \mathrm{H}$ NMR (DMSO- $\left.d_{6}\right) \delta 1.17,1.28\left(2 \times 3 \mathrm{H}, \mathrm{t}, \mathrm{CH}_{2} \underline{\mathrm{CH}}_{3}\right), 4.15,4.24$ (2 x 2H, q, $\left.\mathrm{OCH}_{2} \mathrm{CH}_{3}\right), 5.25\left(2 \mathrm{H}, \mathrm{s}, N-\mathrm{CH}_{2}\right), 7.53(1 \mathrm{H}, \mathrm{s}, 3-\mathrm{H}), 7.93(1 \mathrm{H}, \mathrm{s}, 5-\mathrm{H}), 8.36(1 \mathrm{H}, \mathrm{s}, 6-\mathrm{H}) ;{ }^{13} \mathrm{C}$ NMR $\left(\mathrm{DMSO}-d_{6}\right) \delta 14.0\left(2 \mathrm{x} \mathrm{CH}_{3}\right), 51.0\left(\mathrm{~N}-\mathrm{CH}_{2}\right), 60.7,61.2\left(2 \times \mathrm{OCH}_{2} \mathrm{CH}_{3}\right), 74.6$ (7-C), 114.2, 114.8 (2 x CN), 125.5 (2-C), 137.8 (5-C), 154.6 (6-C), 159.5 (2- $\left.\mathrm{CO}_{2} \mathrm{Et}\right), 167.9$ (1- $\left.\mathrm{CH}_{2} \mathrm{CO}_{2} \mathrm{Et}\right)$; MS (EI) $\mathrm{M}^{+}, m / z$ 301. Anal. Calcd. for $\mathrm{C}_{15} \mathrm{H}_{15} \mathrm{~N}_{3} \mathrm{O}_{4}$ (301.4): C, 59.79; H, 5.02; N, 13.95. Found: C, 59.45; H, 4.74; N, 14.08 .

Dimethyl 4-[5-(alkoxycarbonyl)-1H-pyrrol-3-yl]-2,6-dimethyl-1,4-dihydropyridine-3,5dicarboxylates (9). To a solution of alkyl 4-formyl-1H-pyrrole-2-carboxylate 2 (10 mmol) in methanol $(50 \mathrm{~mL})$ methyl acetoacetate $(2.32 \mathrm{~g}, 20 \mathrm{mmol})$ was added followed by $\mathrm{NH}_{4} \mathrm{OH}(28 \%$ $\mathrm{NH}_{3}$ in water, $2 \mathrm{~mL}, 15 \mathrm{mmol}$ ). The mixture was heated at reflux for $6 \mathrm{~h}$ and then poured into ice water and extracted with $\mathrm{CHCl}_{3}$. The extract was dried $\left(\mathrm{MgSO}_{4}\right)$, concentrated to dryness; the product was isolated by column chromatography (silica gel, $\mathrm{CHCl}_{3}$ ) and recrystallized from a suitable solvent.

Dimethyl 4-[5-(methoxycarbonyl)-1H-pyrrol-3-yl]-2,6-dimethyl-1,4-dihydropyridine- 3,5dicarboxylate (9a). Yield $1.18 \mathrm{~g}(34 \%)$, yellowish crystals, mp $214-6{ }^{\circ} \mathrm{C}$ (methanol). IR v 1707, 1684, $1651(\mathrm{C}=\mathrm{O}) \mathrm{cm}^{-1} ;{ }^{1} \mathrm{H}$ NMR $\left(\mathrm{CDCl}_{3}\right) \delta 2.32(6 \mathrm{H}, \mathrm{s}, 2,6-\mathrm{Me}), 3.69\left(6 \mathrm{H}, \mathrm{s}, 3,5-\mathrm{CO}_{2} \mathrm{Me}\right), 3.80$ $\left(3 \mathrm{H}, \mathrm{s}, 5\right.$ ' $\left.-\mathrm{CO}_{2} \mathrm{Me}\right), 4.96(1 \mathrm{H}, \mathrm{s}, 4-\mathrm{H}), 5.74(1 \mathrm{H}, \mathrm{bs}, 1-\mathrm{H}), 6.70(1 \mathrm{H}, \mathrm{m}, 4$ '- $), 6.75\left(1 \mathrm{H}, \mathrm{m}, 2^{\prime}-\right.$ $\mathrm{H}), 8.86\left(1 \mathrm{H}, \mathrm{bs}, 1^{\prime}-\mathrm{H}\right) ;{ }^{13} \mathrm{C} \mathrm{NMR}\left(\mathrm{CDCl}_{3}\right) \delta 19.5$ (2,6-Me), $31.3(4-\mathrm{C}), 51.1\left(3,5-\mathrm{CO}_{2} \mathrm{Me}\right), 51.3$ (5'- $\left.\mathrm{CO}_{2} \underline{\mathrm{Me}}\right), 103.5$ (3,5-C), 114.0 (4'-C), 120.3 (2'-C), 132.1 (3',5'- C), 144.4 (2,6-C), 161.6 (5'- $\left.{ }^{\prime} \mathrm{O}_{2} \mathrm{Me}\right), 168.0\left(3,5-\underline{\mathrm{CO}}_{2} \mathrm{Me}\right)$. Anal. calcd. for $\mathrm{C}_{17} \mathrm{H}_{20} \mathrm{~N}_{2} \mathrm{O}_{6}(348.4)$ : C, 58.61; H, 5.79; N, 8.04. Found: C, 58.55; H, 5.70; N, 7.89.

Dimethyl 4-[5-(ethoxycarbonyl)-1H-pyrrol-3-yl]-2,6-dimethyl-1,4-dihydropyridine-3,5dicarboxylate (9b). Yield $2.64 \mathrm{~g}(75 \%)$, yellowish crystals, mp $209-11{ }^{\circ} \mathrm{C}$ (ethanol). IR v 1705 , 1680, $1655(\mathrm{C}=\mathrm{O}) \mathrm{cm}^{-1} ;{ }^{1} \mathrm{H} \mathrm{NMR}\left(\mathrm{CDCl}_{3}\right) \delta 1.32\left(3 \mathrm{H}, \mathrm{t}, 5^{\prime}-\mathrm{CO}_{2} \mathrm{CH}_{2} \mathrm{CH}_{3}\right), 2.33(6 \mathrm{H}, \mathrm{s}, 2,6-\mathrm{Me})$, $3.70\left(6 \mathrm{H}, \mathrm{s}, 3,5-\mathrm{CO}_{2} \mathrm{Me}\right), 4.27\left(2 \mathrm{H}, \mathrm{q}, 5{ }^{\prime}-\mathrm{CO}_{2} \mathrm{CH}_{2} \mathrm{CH}_{3}\right), 4.96(1 \mathrm{H}, \mathrm{s}, 4-\mathrm{H}), 5.74(1 \mathrm{H}, \mathrm{bs}, 1-\mathrm{H})$, $6.71\left(2 \mathrm{H}, \mathrm{m}, 2^{\prime}, 4^{\prime}-\mathrm{H}\right), 8.84\left(1 \mathrm{H}, \mathrm{bs}, 1^{\prime}-\mathrm{H}\right) ;{ }^{13} \mathrm{C} \mathrm{NMR}\left(\mathrm{CDCl}_{3}\right) \delta 14.5\left(5^{\prime}-\mathrm{CO}_{2} \mathrm{CH}_{2} \mathrm{CH}_{3}\right), 19.6$ (2,6-Me), 31.3 (4-C), 51.1 (3,5- $\left.\mathrm{CO}_{2} \mathrm{Me}\right), 60.1$ (5'- $\left.\mathrm{CO}_{2} \mathrm{CH}_{2} \mathrm{CH}_{3}\right), 103.5$ (3,5-C), 113.8 (4'-C), 120.1 (2'-C), 132.0 (3',5'- C), 144.3 (2,6-C), 161.3 (5'- $\left.\mathrm{CO}_{2} \mathrm{Et}\right), 168.0$ (3,5- $\left.\mathrm{CO}_{2} \mathrm{Me}\right)$. Anal. Calcd. for $\mathrm{C}_{18} \mathrm{H}_{22} \mathrm{~N}_{2} \mathrm{O}_{6}(362.4)$ : C, 59.66; H, 6.12; N, 7.73. Found: C, 59.52; H, 6.00; N, 7.54. 


\section{Dimethyl 4-[5-(alkoxycarbonyl)-1-(2-alkoxy-2-oxoethyl)-1H-pyrrol-3-yl]-2,6-dimethyl-1,4- dihydropyridine-3,5-dicarboxylates (10).}

Route a: by the general procedure for compounds 9, using of formyl derivative $\mathbf{3 a}$ and $\mathbf{3 b}$, respectively instead of $\mathbf{2 a}$ and $\mathbf{2} \mathbf{b}$.

Route b: by the general method for alkylated derivatives 3 , using of substituted 1,4dihydropyridines 9 (10 $\mathrm{mmol})$ as a substrate and the ratio of dihydropyridine: alkylating agent was $1: 2$ and 1:2.4, respectively. The product was isolated by column chromatography (silica gel, $\mathrm{CHCl}_{3}$ ) and recrystallized from a suitable solvent.

Dimethyl 4-[5-(methoxycarbonyl)-1-(2-methoxy-2-oxoethyl)-1H-pyrrol-3-yl]-2,6-dimethyl1,4-dihydropyridine-3,5-dicarboxylate (10a). Yield $2.2 \mathrm{~g}(52 \%$, route $a), 1.9 \mathrm{~g}(45 \%$, route $b)$, yellow crystals, mp $192-4{ }^{\circ} \mathrm{C}$ (methanol). IR v 1707, 1694, $1651(\mathrm{C}=\mathrm{O}) \mathrm{cm}^{-1} ;{ }^{1} \mathrm{H} \mathrm{NMR}\left(\mathrm{CDCl}_{3}\right)$ $\delta 2.32(6 \mathrm{H}, \mathrm{s}, 2,6-\mathrm{Me}), 3.70\left(6 \mathrm{H}, \mathrm{s}, 3,5-\mathrm{CO}_{2} \mathrm{Me}\right), 3.73\left(3 \mathrm{H}, \mathrm{s}, 1^{\prime}-\mathrm{CH}_{2} \mathrm{CO}_{2} \mathrm{Me}\right), 3.75$ (3H, s, 5'$\left.\mathrm{CO}_{2} \mathrm{Me}\right), 4.90\left(2 \mathrm{H}, \mathrm{s}, 1^{\prime}-\mathrm{CH}_{2} \mathrm{CO}_{2} \mathrm{Me}\right), 4.94(1 \mathrm{H}, \mathrm{s}, 4-\mathrm{H}), 5.72(1 \mathrm{H}, \mathrm{bs}, 1-\mathrm{H}), 6.61(1 \mathrm{H}, \mathrm{d}, J=2.1$, 4'-H), 6.75 (1H, d, $J=2.1,2$ '-H); ${ }^{13} \mathrm{C}$ NMR $\left(\mathrm{CDCl}_{3}\right) \delta 19.3$ (2,6-Me), 31.0 (4-C), 50.4, 50.9 (1'$\left.\mathrm{CH}_{2} \mathrm{CO}_{2} \underline{\mathrm{Me}}, 5^{\prime}-\mathrm{CO}_{2} \underline{\mathrm{Me}}\right), 51.0$ (3,5- $\mathrm{CO}_{2} \underline{\mathrm{Me}}$ ), 52.3 (1' $-\underline{\mathrm{CH}_{2}} \mathrm{CO}_{2} \mathrm{Me}$ ), 102.9 (3,5-C), 117.0 (4'C), 121.4 (5'-C), 127.2 (2'-C), 129.9 (3'- C), 144.9 (2,6-C), $161.6\left(5^{\prime}-\mathrm{CO}_{2} \mathrm{Me}\right), 168.1$ (3,5-

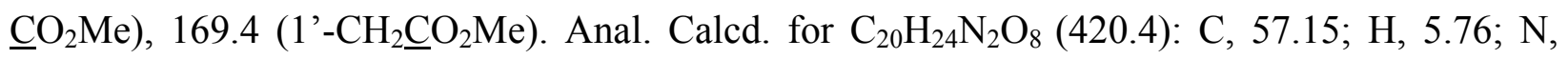
6.66. Found: C, 56.99; H, 5.74; N, 6.62.

Dimethyl 4-[5-(ethoxycarbonyl)-1-(2-ethoxy-2-oxoethyl)-1H-pyrrol-3-yl]-2,6-dimethyl-1,4dihydropyridine-3,5-dicarboxylate (10b). Yield $2.11 \mathrm{~g}(47 \%$, route $a), 1.9 \mathrm{~g}(42 \%$, route $b)$, yellow crystals, mp $148-50{ }^{\circ} \mathrm{C}$ (ethanol). IR v 1738, 1698, $1651(\mathrm{C}=\mathrm{O}) \mathrm{cm}^{-1} ;{ }^{1} \mathrm{H} \mathrm{NMR}\left(\mathrm{CDCl}_{3}\right) \delta$ $1.30\left(6 \mathrm{H}, \mathrm{m}, 1^{\prime}-\mathrm{CH}_{2} \mathrm{CO}_{2} \mathrm{CH}_{2} \underline{\mathrm{CH}}_{3}, 5^{\prime}-\mathrm{CO}_{2} \mathrm{CH}_{2} \underline{\mathrm{CH}}_{3}\right), 2.32(6 \mathrm{H}, \mathrm{s}, 2,6-\mathrm{Me}), 3.70(6 \mathrm{H}, \mathrm{s}, 3,5-$ $\left.\mathrm{CO}_{2} \mathrm{Me}\right), 4.23\left(4 \mathrm{H}, \mathrm{m}, 1^{\prime}-\mathrm{CH}_{2} \mathrm{CO}_{2} \underline{\mathrm{CH}}_{2} \mathrm{CH}_{3}, 5^{\prime}-\mathrm{CO}_{2} \underline{\mathrm{CH}}_{2} \mathrm{CH}_{3}\right), 4.89$ (2H, s, 1' $\left.-\underline{\mathrm{CH}}_{2} \mathrm{CO}_{2} \mathrm{Et}\right), 4.96$ $(1 \mathrm{H}, \mathrm{s}, 4-\mathrm{H}), 5.78(1 \mathrm{H}, \mathrm{bs}, 1-\mathrm{H}), 6.59\left(1 \mathrm{H}, \mathrm{d}, J=2.1,4^{\prime}-\mathrm{H}\right), 6.76\left(1 \mathrm{H}, \mathrm{d}, J=2.1,2^{\prime}-\mathrm{H}\right) ;{ }^{13} \mathrm{C}$ $\operatorname{NMR}\left(\mathrm{CDCl}_{3}\right) \delta 14.3\left(1^{\prime}-\mathrm{CH}_{2} \mathrm{CO}_{2} \mathrm{CH}_{2} \underline{\mathrm{CH}}_{3}, 5^{\prime}-\mathrm{CO}_{2} \mathrm{CH}_{2} \underline{\mathrm{CH}}_{3}\right.$ ), 19.3 (2,6-Me), 31.0 (4-C), 51.0 (3,5- $\left.-\mathrm{CO}_{2} \underline{\mathrm{Me}}\right), 52.3\left(1^{\prime}-\underline{\mathrm{CH}_{2}} \mathrm{CO}_{2} \mathrm{Et}\right), 59.8\left(1^{\prime}-\mathrm{CH}_{2} \mathrm{CO}_{2} \underline{\mathrm{CH}_{2}} \mathrm{CH}_{3}, 5^{\prime}-\mathrm{CO}_{2} \underline{\mathrm{CH}}_{2} \mathrm{CH}_{3}\right), 102.9,103.2$ (3,5-C), 116.8 (4'-C), 121.8 (5'-C), 127.1 (2'-C), 129.8 (3'- C), 144.9 (2,6-C), 161.2 (5'$\left.\mathrm{CO}_{2} \mathrm{Et}\right), 168.1\left(3,5-\underline{\mathrm{CO}}_{2} \mathrm{Me}\right), 169.4\left(1 '-\mathrm{CH}_{2} \underline{\mathrm{CO}}_{2} \mathrm{Et}\right)$. Anal. Calcd. for $\mathrm{C}_{22} \mathrm{H}_{28} \mathrm{~N}_{2} \mathrm{O}_{8}$ (448.5): C, 58.92; H, 6.29; N, 6.25. Found: C, 58.81; H, 6.18; N, 6.17.

Dimethyl 4-[5-(methoxycarbonyl)-1H-pyrrol-3-yl]-1-(2-methoxy-2-oxoethyl)-2,6-dimethyl1,4-dihydropyridine-3,5-dicarboxylate (11). Sodium methoxide (0.54 g, $10 \mathrm{mmol})$, methyl acetoacetate $(2.32 \mathrm{~g}, 20 \mathrm{mmol})$ and methyl 4-formyl-1H-pyrrole-2-carboxylate (2a, $1.53 \mathrm{~g}$, $10 \mathrm{mmol})$ were added to a solution of glycine methyl ester hydrochloride $(1.26 \mathrm{~g}, 10 \mathrm{mmol})$ in methanol $(50 \mathrm{~mL})$. The reaction mixture was refluxed for $10 \mathrm{~h}$, then evaporated to dryness and purified on a silica gel column using $\mathrm{CHCl}_{3}$. After distilling off the solvent, the product was obtained as a yellow solid, yield $1.89 \mathrm{~g}(45 \%), \mathrm{mp} 57-60{ }^{\circ} \mathrm{C}$ (methanol). ${ }^{1} \mathrm{H} \mathrm{NMR}\left(\mathrm{CDCl}_{3}\right) \delta$ $2.39(6 \mathrm{H}, \mathrm{s}, 2,6-\mathrm{Me}), 3.72\left(6 \mathrm{H}, \mathrm{s}, 3,5-\mathrm{CO}_{2} \mathrm{Me}\right), 3.74\left(3 \mathrm{H}, \mathrm{s}, 1-\mathrm{CH}_{2} \mathrm{CO}_{2} \underline{\mathrm{Me}}\right), 3.77$ (3H, s, 5'$\left.\mathrm{CO}_{2} \mathrm{Me}\right), 4.33\left(2 \mathrm{H}, \mathrm{s}, 1-\underline{\mathrm{CH}}_{2} \mathrm{CO}_{2} \mathrm{Me}\right), 4.94(1 \mathrm{H}, \mathrm{s}, 4-\mathrm{H}), 5.97(1 \mathrm{H}, \mathrm{bs}, 1$ ' $-\mathrm{H}), 6.72$ (2H, m, 2', 4' $\mathrm{H}) ;{ }^{13} \mathrm{C} \mathrm{NMR}\left(\mathrm{CDCl}_{3}\right) \delta 16.2(2,6-\mathrm{Me}), 31.2(4-\mathrm{C}), 51.2\left(3,5-\mathrm{CO}_{2} \mathrm{Me}\right), 51.4\left(1-\mathrm{CH}_{2} \mathrm{CO}_{2} \underline{\mathrm{Me}}, 5^{\prime}-\right.$ $\mathrm{CO}_{2} \underline{\mathrm{Me}}$ ), 52.2 (1- $\left.\underline{\mathrm{CH}}_{2} \mathrm{CO}_{2} \mathrm{Me}\right), 107.3$ (3,5-C), 114.0 (4'-C), 120.7 (2'-C), 131.0 (3',5'-C), 144.0 
(2,6-C), $161.5\left(5^{\prime}-\mathrm{CO}_{2} \mathrm{Me}\right), 168.2\left(3,5-\underline{\mathrm{CO}}_{2} \mathrm{Me}\right), 169.9$ (1- $\left.\mathrm{CH}_{2} \mathrm{CO}_{2} \mathrm{Me}\right)$. Anal. Calcd. for $\mathrm{C}_{20} \mathrm{H}_{24}$ $\mathrm{N}_{2} \mathrm{O}_{8}$ (420.4): C, 57.15; H, 5.76; N, 6.66. Found: C, 57.01; H, 5.67; N, 6.52.

Dimethyl 4-[5-(methoxycarbonyl)-1-(2-methoxy-2-oxoethyl)-1H-pyrrol-3-yl]-1-(2-methoxy-2-oxoethyl)-2,6-dimethyl-1,4-dihydropyridine-3,5-dicarboxylate (12).

Route c: By the previous procedure for the compound 11, using the formyl derivative 3a $(10 \mathrm{mmol})$ instead of $\mathbf{2 a}$. Yield $2.12 \mathrm{~g}(43 \%)$, yellow crystals, mp $52-5{ }^{\circ} \mathrm{C}$ (methanol).

Route d: The mixture of dimethyl 4-[5-(methoxycarbonyl)-1-(2-methoxy-2-oxoethyl)-1Hpyrrol-3-yl]-2,6- dimethyl-1,4-dihydropyridine-3,5-dicarboxylate (10a, $2.10 \mathrm{~g}, 5 \mathrm{mmol}$ ), solid $\mathrm{K}_{2} \mathrm{CO}_{3}(1.38 \mathrm{~g}, 10 \mathrm{mmol})$, Aliquat 336 (4.04 g, $\left.10 \mathrm{mmol}\right)$, and methyl bromoacetate $(6.5 \mathrm{~g}$, $16 \mathrm{mmol}$ ) was stirred and heated in closed tube at $200{ }^{\circ} \mathrm{C}$ for 4 days. After cooling, the reaction mixture was concentrated and chromatographed on silica gel (eluent $\mathrm{CHCl}_{3}$ ). Yield $0.86 \mathrm{~g}$ (35\%), mp 52-5 ${ }^{\circ} \mathrm{C} .{ }^{1} \mathrm{H} \mathrm{NMR}\left(\mathrm{CDCl}_{3}\right) \delta 2.39(6 \mathrm{H}, \mathrm{s}, 2,6-\mathrm{Me}), 3.72\left(6 \mathrm{H}, \mathrm{s}, 3,5-\mathrm{CO}_{2} \mathrm{Me}\right), 3.73$ $\left(6 \mathrm{H}, \mathrm{s}, 1,1^{\prime}-\mathrm{CH}_{2} \mathrm{CO}_{2} \mathrm{Me}\right), 3.76\left(3 \mathrm{H}, \mathrm{s}, 5^{\prime}-\mathrm{CO}_{2} \mathrm{Me}\right), 4.34\left(2 \mathrm{H}, \mathrm{s}, 1-\underline{\mathrm{CH}}_{2} \mathrm{CO}_{2} \mathrm{Me}\right), 4.91(3 \mathrm{H}, \mathrm{s}, 4-\mathrm{H}$, $\left.1^{\prime}-\underline{\mathrm{CH}}_{2} \mathrm{CO}_{2} \mathrm{Me}\right), 6.66\left(1 \mathrm{H}, \mathrm{m}, 4\right.$ '-H), $6.74\left(1 \mathrm{H}, \mathrm{m}, 2\right.$ ' $-\mathrm{H} ;{ }^{13} \mathrm{C} \mathrm{NMR}\left(\mathrm{CDCl}_{3}\right) \delta 15.7$ (2,6-Me), 30.6 (4-C), 50.5, 50.9 (1,1'- $\mathrm{CH}_{2} \mathrm{CO}_{2} \underline{\mathrm{Me}}, 5$ ' $\left.-\mathrm{CO}_{2} \underline{\mathrm{Me}}\right), 51.8$ (3,5- $\left.\mathrm{CO}_{2} \underline{\mathrm{Me}}\right), 52.2$ (1, ' $\left.-\underline{\mathrm{CH}_{2}} \mathrm{CO}_{2} \mathrm{Me}\right)$, 106.6 (3,5-C), 116.4 (4'-C), 121.0 (5'ㄷ), 128.3 (2',3'-C), 141.1 (2,6-C), 161.1 (5'- CO $\left._{2} \mathrm{Me}\right)$, 167.7 (3,5- $\left.\underline{-} \mathrm{CO}_{2} \mathrm{Me}\right), 169.5\left(1,1\right.$ ' $\left.-\mathrm{CH}_{2} \mathrm{CO}_{2} \mathrm{Me}\right)$. Anal. Calcd. for $\mathrm{C}_{23} \mathrm{H}_{28} \mathrm{~N}_{2} \mathrm{O}_{10}$ (492.5): C, 56.61; H, 5.73; N, 5.69. Found: C, 56.31; H, 5.52; N, 5.40.

\section{Acknowledgements}

The authors would like to thank Slovak Grant Agency (financial support No.1/0058/03, 1/2448/05) and Agency for Science and Technique (financial support No. 20-007304) as well as Dr. Eva Solčániová (Institute of Chemistry, Comenius University, Bratislava, Slovak Republic) for some NMR measurements, and valuable discussions.

\section{References and Notes}

1. (a) Massa, S.; Di Santo, R.; Mai, A.; Botta, M.; Artico, M.; Panico, S.; Simonetti, G. Farmaco 1990, 45, 833. (b) Bijev, A.; Radev, I.; Borisova, I. Pharmazie 2000, 55, 568. (c) Bijev, A.; Hung, V. Arzneim. Forsch. 2001, 51, 667.

2. Komiyama, K.; Tronquet, C.; Hirokawa, Y.; Funayama, S.; Satoh, O.; Umezawa, I.; Oishi, S. Jpn. J. Antibiot. 1986, 39, 746.

3. (a) Kuroda, Y.; Okuhara, M.; Goto, T.; Okamoto, M.; Yamashita, M; Kohsaka, M.; Aoki, H.; Imanaka, H. J. Antibiotic (Tokyo) 1980, 33, 267. (b) Westley, J. W.; Evans, R. H.; Sello, L. H.; Troupe, N.; Liu, C. M.; Blount, J. F. J. Antibiot. (Tokyo) 1979, 32, 100. (c) Penco, S.; Redaelli, S.; Arcamone, F. Gazz. Chim. Ital. 1967, 97, 110. (d) Koyama, M.; Kodama, Y.; 
Tsuruoka, T.; Ezaki, N.; Niwa, T.; Inouye, S. J. Antibiot. (Tokyo) 1981, 34, 1569. (e) Kaneda, M.; Nakamura, S.; Ezaki, N.; Iitaka, Y. J. Antibiot. (Tokyo) 1981, 34, 1366.

4. Baraldi, P. G.; Beria, I.; Cozzi, P.; Bianchi, N.; Gambari, R.; Romagnoli, R. Bioorganic \& Medicinal Chemistry 2003, 11, 965.

5. (a) Langner, A.; Rätzer, J.; Rickert, H.; Nerlich, C.; Franke, P. Pharmazie 1993, 48, 849. (b) Rostock, A.; Tober, C.; Rundfeldt, C.; Bartsch, R.; Unverferth, K.; Engel, J. V.; White, H. S. Epilepsy Res. 1997, 28, 17.

6. Sundberg, R. J. In Comprehensive Heterocyclic Chemistry; Pergamon Press: 1984; Vol. 4, Part 3, pp 370-376.

7. Wierzbicki, M.; Bonnet, J.; Tsouderos, Y. Eur. Pat. Appl. EP 1990, 349,432; Chem. Abstr. 1990, 113, 23686u.

8. (a) Trofimov, B. A. In The Chemistry of Heterocyclic Compounds. 48. Pyrroles, Part 2; Wiley: New York, 1992; pp 131-298. (b) Trofimov, B. A.; Sobenina, L. N.; Demenev, A. P.; Mikhaleva, A. I. Chem. Rev. 2004, 104, 2481.

9. Harbuck, J. W.; Rapoport, H. J. Org. Chem. 1972, 37, 3618.

10. Bailey, D. M.; Johnson, R. E.; Albertson, N. F. Organic Syntheses, Coll. 1988; Vol. 6, pp 618-619.

11. Sonnet, P. E. J. Med. Chem. 1972, 15, 97.

12. Fournari, P.; Farnier, M.; Fournier, C. Bull. Soc. Chim. Fr. 1972, 283.

13. Lokaj, J.; Kettmann, V.; Milata, V.; Štetinová J.; Petrov, O. Acta Cryst. 2001, E57, 404.

14. Abraham, R. J.; Lapper, R. D.; Smith, K. M.; Unsworth, J. F. J. Chem. Soc., Perkin Trans 2 1974, 1004.

15. (a) Sobenina, L. N.; Demenev, A. P.; Mikhaleva, A. I.; Trofimov, B. A. Usp. Khim. 2002, 71, 641. (b) Sha, C. K.; Liu, J. M.; Chiang, R. K.; Wang, S. L. Heterocycles 1990, 31, 603.

(c) Kada, R.; Ilavský, D.; Goljer, I.; Gahér, P. Collect. Czech. Chem. Commun. 1991, 56, 418. (d) Haubmann, C.; Hübner, H.; Gemeiner, P. Bioorganic Med. Chem. Lett. 1999, 9, 3143.

16. Breslow, D. S.; Baumgarten, E.; Hauser, C. R. J. Am. Chem. Soc. 1944, 66, 1286.

17. Sakamoto, M.; Nozaka, A.; Shimamoto, M.; Ozaki, H.; Suzuki, Y.; Yoshioka, S.; Nagano, M.; Okamura, K.; Date, T.; Tamura, O. J. Chem. Soc., Perkin Trans 1, 1995, 1759.

18. Kambe, S.; Saito, K.; Makano, Y. Synthesis 1981, 212,123.

19. Copeland, R. A. B.; Day, A. R. J. Amer. Chem. Soc. 1943, 65, 1072.

20. (a) Štetinová, J.; Kada, R.; Leško, J.; Zalibera, L.; Ilavský, D.; Bartovič, A. Collect. Czech. Chem. Commun. 1995, 60, 999. (b) Štetinová, J.; Kada, R.; Leško, J.; Dandárová, M.; Krublová, M. Collect. Czech. Chem. Commun. 1996, 61, 921.

21. Lokaj, J.; Kettmann, V.; Štetinová, J.; Kottas, P. Chem. Pap. 2002, 56, 127.

22. (a) Eisner, U.; Kuthan, J. Chem. Rev. 1972, 72, 1. (b) Stout, D. M.; Meyers, A. I. Chem. Rev. 1982, 82, 223. (c) Sausinš, A.; Duburs, G. Heterocycles 1988, 27, 269, 291.

23. Loev, B.; Goodman, M. M.; Snader, K. M.; Tadeschi, R.; Macko, E. J. Med. Chem. 1974, 17, 956. 
24. Hantzsch, A. Liebigs Ann. 1882, $215,1$.

25. Iwanami, M.; Shibanuma, T.; Fujimoto, M.; Kawai, R.; Tamayawa, K.; Takenaka, T.; Takahashi, K.; Murakami, M. Chem. Pharm. Bull. 1979, 27, 1426.

26. Barry, J.; Bram, G.; Petit, A. Tetrahedron Lett. 1988, 29, 4567.

27. Kurfürst, A.; Trška, P.; Goljer, I. Collection Czechoslovak Chem. Commun. 1984, 49, 2393.

28. The compound did not analyze correctly. 